\title{
FATORES MOTIVACIONAIS DE IDOSOS PARTICIPANTES EM PROJETO SOCIAL UNIVERSITÁRIO DE EDUCAÇÃO FISICA
}

\author{
Adriana Schüler Cavalli ${ }^{1}$ \\ Giulia Pinheiro Garcia² \\ Amine Cabral Ricardo ${ }^{3}$ \\ José Antonio Bicca Ribeiro ${ }^{4}$ \\ Marcelo Olivera Cavalli ${ }^{5}$
}

resumo

Objetivo: o presente estudo teve por objetivo investigar os fatores de motivação de idosos para a prática de atividade física. Método: trata-se de um estudo descritivo transversal realizado com 35 idosos com 10 anos ou mais de participação em um projeto social de extensão universitária. A coleta dos dados se deu por meio de entrevistas individuais referentes a aspectos sócio-demográficos,

\footnotetext{
1 Graduada em Educação Física. Doutora em Ciências da Saúde e Esporte pela Chukyo University. Professora Associada da Universidade Federal de Pelotas (UFPel). E-mail: adriscavalli@gmail.com.

2 Graduada em Educação Física pela Universidade Federal de Pelotas (UFPel).

3 Graduada em Educação Física pela Universidade Federal de Pelotas (UFPel).

4 Graduado em Educação Física. Mestre em Educação Física pela Universidade Federal de Pelotas (UFPel). Professor Substituto da Escola Superior de Educação Física da UFPel.

5 Graduado em Educação Física. Doutor em Ciências da Saúde e Esporte pela Chukyo University. Professor Associado da Universidade Federal de Pelotas (UFPel).
} 
prática de atividade física e motivos de participação no projeto. Para a mensuração da motivação foi utilizado o Inventário de Motivação à Prática Regular de Atividade Física 54 (IMPRAF 54). Resultados: a amostra foi composta em sua maioria por mulheres ( $n=33,94 \%)$ com média de idade de $\pm 74,05(\mathrm{DP} \pm 6,30$ ) anos. Os resultados indicaram que os idosos continuam motivados a praticar atividade física principalmente pela saúde, seguida pelo prazer e pela sociabilidade. Conclusão: quanto à importância que dão à prática de atividade física, foi identificado que os idosos conhecem seus benefícios, o que faz com que permaneçam no programa. Os idosos afirmaram que a prática de atividade física está relacionada à prevenção de doenças e/ou manutenção da saúde, demonstrando que a atividade física, na concepção deles, está ligada principalmente à saúde.

palavras-chave

Motivação. Idoso. Envelhecimento. Atividade Motora. Promoção da Saúde.

\section{Introdução}

O envelhecimento populacional é um dos grandes desafios enfrentados pela humanidade na atualidade. No Brasil, a proporção de pessoas com 60 anos ou mais está crescendo mais rapidamente do que a das demais faixas etárias. Em termos mundiais, entre 1970 e 2025, espera-se um aumento de 223\% da população idosa. Isso representa um aumento de aproximadamente 694 milhões de pessoas mais velhas. A estimativa da população idosa para 2025 é de aproximadamente 1,2 bilhão de pessoas com 60 ou mais anos de idade. Até 2050 deverá ser de 2 bilhões, sendo 80\% destes nos países em desenvolvimento (OPAS, 2005).

O processo de envelhecimento é acompanhado, muitas vezes, por um estilo de vida inativo, que favorece uma diminuição da capacidade física e a dependência. Pesquisa realizada pelo Ministério da Saúde (MS, 2007) nas capitais brasileiras determinou que a inatividade física ocorre em grande parte da população idosa. Os resultados do estudo indicam percentuais de 50,3\% para mulheres e $65,4 \%$ para homens acima dos 65 anos de idade. King (2001) relata que somente uma reduzida parcela de indivíduos idosos nos Estados Unidos e demais países industrializados realiza atividade física regular e em nível recomendado. Essas constatações confirmam que o contexto do envelhecimento não é uma exclusividade do Brasil. 
Diante do fenômeno do crescimento vertiginoso no número de idosos no Brasil associado a um estilo de vida sedentário decorrem preocupações nas mais diferentes áreas da vida e do conhecimento humano (SIQUEIRA et al., 2009). No que se refere ao dia a dia, questões previdenciárias têm causado inquietação nos setores da economia e no planejamento dos trabalhadores. Em termos acadêmicos relacionados à saúde do idoso, tem-se procurado investigar e fomentar infraestruturas e métodos para favorecer um envelhecimento mais saudável e com qualidade.

A literatura científica específica já comprovou que quanto mais ativo fisicamente a pessoa for, menor será o risco que ela tem de apresentar limitações físicas. Dentre os vários benefícios que a prática de atividade física pode acarretar, um dos principais é a manutenção da capacidade funcional compreendida como a capacidade de realizar as atividades práticas do dia a dia (FRANCHI; MONTENEGRO JUNIOR, 2005; WHO, 2010).

A Sociedade Brasileira de Geriatria e Gerontologia (SBGG) afirma que a atividade física aliada a uma dieta equilibrada, ao controle de peso e hábitos de vida saudáveis pode melhorar a qualidade de vida de idosos. A SBGG ressalta, ainda, que a prática de atividades físicas auxilia na melhora da autoimagem, da autoconfiança, da afetividade e da sociabilização de idosos. Assim, pode-se compreender que os benefícios das atividades físicas se fazem presentes nos aspectos biológicos, psicológicos e sociais de idosos (MAZO; LOPES; BENEDETTI, 2009).

Conforme apontado por Freitas et al. (2007), a busca pela prática de atividade física em programas voltados para a promoção de saúde vem crescendo. Contudo, a procura pelos indivíduos idosos ainda é menor quando comparada com indivíduos mais jovens. Por esse motivo, reconhecer a importância da atividade física para a saúde e a qualidade de vida de um indivíduo é extremamente importante. Nesse sentido, se faz necessário determinar os fatores que motivam os indivíduos a praticarem atividade física regularmente (COSTA et al., 2003).

A motivação, de acordo com Samulski (2002), pode ser caracterizada como um processo ativo, intencional e dirigido a uma meta, o qual depende da interação de fatores pessoais (intrínsecos) e ambientais (extrínsecos). Importante salientar que a adoção de um estilo de vida ativo requer disciplina, para que o indivíduo dê continuidade à tarefa. A mudança de comportamento deve ser relevante em sua vida, sendo necessário, assim, que o indivíduo mantenha essa atitude.

A mudança de comportamento para a prática de atividade física é respaldada por teorias e modelos. O modelo transteorético, desenvolvido por Prochaska, DiClemente e Norcross (1992), descreve cinco diferentes estágios envolvidos na aquisição ou manutenção de um comportamento ativo à medida que o indivíduo avalia se o mesmo é benéfico para a sua vida e o mantém (DUMITH; GIGANTE; DOMINGUES, 2007). 
Para tanto, é importante ressaltar a imprescindibilidade de profissionais especializados, qualificados e competentes para atender e "cativar" a população idosa, demonstrando que o domínio profissional vai além das questões técnicas. É imperativo desenvolver um trabalho psicológico, físico e social integrado, procurando motivar os idosos para que a prática se torne algo prazeroso, contínuo e benéfico.

Conhecendo os benefícios decorrentes da prática contínua e orientada de atividades físicas, torna-se primordial reconhecer os fatores que influenciam e motivam os idosos a se inserirem e manterem um estilo de vida ativo e saudável. Desta maneira, o presente estudo se propôs a investigar os fatores de motivação de idosos participantes por pelo menos dez anos de um projeto social universitário que visa o envelhecimento saudável por meio da atividade física.

\section{Método}

Este estudo tem um caráter descritivo transversal (THOMAS; NELSON; SILVERMAN, 2009). A amostra foi intencional e composta por idosos que participavam de um projeto de extensão universitária de atividades físicas e de lazer.

O referido projeto foi estabelecido em 1993 e tem atendido idosos desde então, com o intuito de proporcionar um envelhecimento saudável, além de proporcionar a prática profissional dos acadêmicos do curso de Educação Física, preparando-os para o mercado de trabalho (AFONSO et al., 2010). O projeto trabalhava em proporções maiores, e seu carro-chefe sempre foi a hidroginástica por ser uma das únicas instituições na cidade que oferecia esta modalidade gratuitamente. Entretanto, no ano de 2016 houve uma reestruturação institucional e ocorreu a perda do acesso às piscinas, não sendo mais ofertada esta modalidade no projeto. Sendo assim, o projeto deixou de atender cerca de 200 idosos, que em parte poderiam ter participado do presente estudo. Em 2017, cerca de 150 idosos realizavam atividades nas modalidades de ginástica, musculação e treinamento funcional.

Para compor a amostra os idosos deveriam estar vinculados e praticantes há, pelo menos, dez anos no projeto. Outros critérios de inclusão adotados se referiram a: ter 60 anos ou mais, estar engajado no projeto no ano de 2017, estar presente no dia da coleta de dados e aceitar voluntariamente a participar do estudo, por meio da assinatura do Termo de Consentimento de Livre Esclarecido (TCLE).

Para a caracterização sociodemográfica da amostra - idade, sexo, estado civil, escolaridade, doenças, medicamentos - foi realizada uma anamnese. 
$\mathrm{Na}$ anamnese foram incluídas, ainda, questões referentes à importância dada à prática de atividade física e motivos de participação no projeto.

Para a mensuração da motivação foi utilizado o Inventário de Motivação à Prática Regular de Atividade Física 54 (IMPRAF 54) (BARBOSA; BALBINOTTI, 2006). O IMPRAF 54 tem a proposição de medir seis dimensões: Controle de Estresse (CE); Saúde (AS); Sociabilidade (SO); Competitividade (CO); Estética (ES); e Prazer (PR), associadas à motivação para a prática regular de atividades físicas. Visando a identificação das dimensões consideradas mais motivadoras para a prática de atividade física, o instrumento é composto de uma escala Likert de cinco pontos, podendo as respostas ser de "1) Isto me motiva pouquíssimo" a "5) Isto me motiva muitíssimo". A soma dos valores atribuídos para cada fator constitui um escore de motivação que pode variar de 8 a 40. Para contextualizar os resultados, foram utilizadas tabelas normativas, fornecidas no manual de aplicação do instrumento.

Os dados coletados foram colocados em um banco de dados e digitados no programa Excel 2010. A normalidade dos dados foi verificada pelo teste de Shapiro-Wilk, sendo que os mesmos não tiveram distribuição normal, onde se optou pela estatística não paramétrica. Nas comparações entre as variáveis demográficas e de adesão ao projeto foram utilizados os testes de Kruska Wallis e Mann-Whitney. Para todos os testes foi adotada uma significância de 5\%. $\mathrm{Na}$ exposição dos dados foram utilizados valores absolutos e relativos, com valores secundários de médias e desvio padrão.

\section{Resultados}

A amostra foi composta por 35 idosos sendo composta majoritariamente por mulheres $(\mathrm{n}=33,94 \%)$ e dois homens $(\mathrm{n}=2,6 \%)$, com média de idade de $\pm 74,05$ (DP $\pm 6,30$ ) anos. A Tabela 1 , abaixo, expressa os dados sociodemográficos determinados.

Com relação à faixa etária, a maioria dos idosos $(\mathrm{n}=21)$ se encontra com idades entre 70 e 79 anos (60\%); sete (20\%) estão na faixa entre 60 e 69 anos; e os demais sete $(20 \%)$ com 80 ou mais anos. No que se refere à escolaridade, $68 \%$ $(\mathrm{n}=24)$ da amostra tem o Ensino Fundamental completo ou incompleto; $26 \%$ $(n=9)$ tem o Ensino Médio; e somente 6\% $(n=2)$ completou o Ensino Superior.

No que tange à ocupação, a maioria dos idosos $(n=32,91,4 \%)$ é aposentada. No quesito etnia, $91 \%(\mathrm{n}=30)$ da amostra são caucasianos. Em relação ao estado civil, $40 \%(\mathrm{n}=14)$ dos idosos declararam serem viúvos, enquanto que $34 \%(n=12)$ são casados. Ainda pode ser observado que a maioria $(60 \%, n=21)$ 
dos entrevistados vive em companhia de outras pessoas. A respeito do nível socioeconômico, a grande maioria $(\mathrm{n}=29,83 \%)$ ganha dois ou mais salários mínimos regionais, e somente $17 \%(\mathrm{n}=6)$ ganha até um salário mínimo regional.

Tabela 1 - Caracterização sociodemográfica da amostra.

\begin{tabular}{|c|c|c|}
\hline VARIÁVEL & $\mathrm{n}$ & $\%$ \\
\hline \multicolumn{3}{|l|}{ Sexo } \\
\hline Feminino & 33 & 94,0 \\
\hline Masculino & 2 & 6,0 \\
\hline \multicolumn{3}{|l|}{ Faixa Etária } \\
\hline 60 a 69 anos & 7 & 20,0 \\
\hline 70 a 79 anos & 21 & 60,0 \\
\hline 80 anos ou mais & 7 & 20,0 \\
\hline \multicolumn{3}{|l|}{ Escolaridade } \\
\hline Ensino Fundamental* & 24 & 68,0 \\
\hline Ensino Médio* & 9 & 26,0 \\
\hline Ensino Superior ${ }^{\star}$ & 2 & 6,0 \\
\hline \multicolumn{3}{|l|}{ Ocupação } \\
\hline Aposentado & 32 & 91,4 \\
\hline Pensionista & 3 & 8,6 \\
\hline \multicolumn{3}{|l|}{ Cor da Pele } \\
\hline Branca & 30 & 91,0 \\
\hline Não Branca & 5 & 9,0 \\
\hline \multicolumn{3}{|l|}{ Estado Civil } \\
\hline Casado & 12 & 34,0 \\
\hline Viúvo & 14 & 40,0 \\
\hline Outro & 9 & 26,0 \\
\hline \multicolumn{3}{|l|}{ Vive com } \\
\hline Sozinho & 14 & 40,0 \\
\hline Acompanhado & 21 & 60,0 \\
\hline \multicolumn{3}{|l|}{ Renda Média Mensal ${ }^{\star \star}$} \\
\hline Um salário mínimo & 6 & 17,0 \\
\hline Dois salários ou mais & 29 & 83,0 \\
\hline
\end{tabular}

${ }^{*}$ Completo ou incompleto. ${ }^{*}$ Considerando o salário mínimo regional do RS em R\$ 1.103,66. Fonte: Elaborada pelos autores, 2017. 
Na Tabela 2 encontram-se os resultados relacionados às variáveis da condição de saúde dos idosos. Com relação às doenças, foi verificado que $51,0 \%(n=18)$ responderam que possuem duas ou mais doenças, e entre as mais citadas a hipertensão acomete 23 idosos (66\%) e a diabetes 5 deles. Todos os idosos ingerem pelo menos um medicamento ao dia, sendo que a grande maioria da amostra $(83 \%, \mathrm{n}=29)$ relatou tomar dois ou mais medicamentos.

No que diz respeito à percepção subjetiva da condição de saúde, os idosos tem uma percepção positiva, visto que $68,6 \%(n=24)$ consideram ter uma boa e/ou ótima condição. Apenas dois indivíduos percebem sua condição como ruim $(6 \%, n=2)$.

Tabela 2 - Caracterização das variáveis das condições de saúde dos idosos.

\begin{tabular}{lcc}
\hline VARIÁVEL & $\mathrm{n}$ & $\%$ \\
\hline Número de Doenças & 17 & 49,0 \\
Até uma doença & 18 & 51,0 \\
$\quad$ Duas doenças ou mais & & \\
Número de Medicamentos Consumidos & 6 & 17,0 \\
Um medicamento & 29 & 83,0 \\
Dois medicamentos ou mais & & \\
Condição de Saúde & 5 & 14,3 \\
Ótima & 19 & 54,3 \\
Boa & 9 & 25,7 \\
Regular & 2 & 5,7 \\
Ruim &
\end{tabular}

Fonte: Elaborada pelos autores, 2017.

Quando questionados acerca da importância da prática de atividade física em suas vidas, houve um consenso e todos os 35 idosos consideraram fundamental e indispensável a prática de atividade física. Entre os motivos mais citados encontram-se: "prevenir problemas de saúde" e/ou "manter-se saudável" ( $(\mathrm{n}=29)$; "passar o tempo com os amigos" e/ou "ajudar a superar o estresse/diminuir a tensão" ( $n=17)$; nove dos entrevistados citaram o quesito "beleza/estética"; e um deles relatou que durante a atividade física tinha "uma hora para si mesmo".

Quando questionados sobre a possibilidade de desistência de participação no projeto, apenas uma entrevistada disse ter pensado em desistir por se sentir desmotivada após um tempo de afastamento devido a uma fratura. 
Os motivos relatados que os instigaram a participar do projeto por todo o tempo de 10 anos ou mais e se manterem no mesmo foram: "projeto coordenado e organizado por professores e alunos da universidade" $(\mathrm{n}=23)$, "gosto dos amigos" ( $\mathrm{n}=19)$, "gosto do local" $(\mathrm{n}=19)$, ser um projeto gratuito à comunidade $(n=13)$. A divulgação do projeto "boca a boca" foi citada como a razão da grande maioria dos idosos saber da existência do mesmo (89\%).

A Tabela 3 apresenta as características motivacionais dos entrevistados nas seis dimensões do IMPRAF-54. "Saúde" foi determinada como a dimensão que apresentou o maior índice de motivação para a prática de atividade física $(41,57 \pm 4,1)$. As seguintes dimensões foram "Prazer" $(39,57 \pm 4,9)$, "Sociabilidade" (39,37 $\pm 5,7)$ e "Controle de Estresse" (36,08 $\pm 6,6)$. "Estética" (33,02 $\pm 9,0)$, seguida por "Competitividade" $(13,37 \pm 5,3)$, encontram-se como as últimas dimensões a motivar os idosos a praticarem atividade física regular.

Tabela 3 - Escores de motivação apresentadas pelos idosos nas seis dimensões do IMPRAF-54.

\begin{tabular}{lcc}
\hline Dimensão Motivacional & Média (DP) & IC (95\%) \\
\hline Controle do Estresse & $36,08(6,6)$ & 33,79 a 38,37 \\
Saúde & $41,57(4,1)$ & 40,13 a 43,00 \\
Sociabilidade & $39,37(5,7)$ & 37,39 a 41,34 \\
Competitividade & $13,37(5,3)$ & 11,53 a 15,20 \\
Estética & $33,02(9,0)$ & 29,91 a 36,14 \\
Prazer & $39,57(4,9)$ & 37,86 a 41,27 \\
Escore Geral & $203(25,5)$ & 194,31 a 211,68 \\
\hline
\end{tabular}

Legenda: DP = desvio padrão; IC = intervalo de confiança.

Fonte: Elaborada pelos autores, 2017.

Quanto aos resultados dos fatores motivacionais analisados em relação à percepção de saúde, número de doenças e de medicamentos, idade e escolaridade, somente a quantidade de medicamentos foi estatisticamente significante, conforme Tabela 4, abaixo. O que corrobora com os escores das dimensões de motivação onde a saúde mostrou a maior motivação para a prática de atividade física dos idosos, como demonstrado na Tabela 3, acima. 





De acordo com os resultados encontrados neste estudo, "Saúde" é a principal dimensão motivadora para a prática, seguida das dimensões "Prazer", "Sociabilidade" e "Controle de Estresse". Meurer, Benedetti e Mazo (2012), em estudo realizado com 140 idosos com mais de 60 anos participantes de um programa de atividade física por meio da modalidade de ginástica, utilizando o mesmo IMPRAF-54, encontraram resultados que corroboram a mesma ordem dos fatores motivacionais do presente estudo. No estudo de Cardoso (2014), composto por 57 idosos com idade superior a 60 anos participantes de programa de exercício físico generalizado e exercícios aeróbios, foi aplicado o mesmo inventário, e as dimensões "Saúde", "Sociabilidade" e "Prazer" obtiveram os maiores indicadores de motivação.

Cavalli et al. (2014) conduziram um estudo que comparou os fatores motivacionais para prática de atividade física entre idosos brasileiros e portugueses que praticavam ginástica, musculação e hidroginástica. Com amostras predominantemente femininas nos dois países, a saúde foi percebida como uma preocupação comum para ambas as realidades culturais.

O estudo de Loureiro (2007) identificou os principais fatores que motivavam idosos, 50 sujeitos voluntários (14 homens e 36 mulheres) que praticavam atividade física regular na cidade de Porto Alegre/RS, com idade superior a 60 anos. Nos resultados foi ressaltado que o principal motivo para a prática de AF foi a busca pela qualidade de vida, seguido pela manutenção da saúde. Ribeiro et al. (2012) também buscou verificar o que leva os idosos a aderirem a programas de AF e determinar a importância da AF na vida destas pessoas. Os resultados obtidos nesse estudo evidenciaram que o principal motivo para a adesão em um programa de AF é a busca por saúde, seja por interesse próprio, indicação médica e recuperação de lesão. Alguns fatores com caráter psicológico ou social também foram citados, porém com menor importância.

Em outro estudo, realizado por Freitas (2007), na cidade do Recife/PE com 120 idosos de programas de saúde ligados à rede pública avaliados por um questionário adaptado sobre motivação para a prática esportiva, foi observado que fatores relacionados à saúde e ao desempenho físico foram os fatores motivacionais mais relatados entre os idosos, tanto para adesão como para a permanência na prática de atividade física.

Desta forma, os estudos aqui apresentados corroboram com os resultados encontrados no presente estudo, demostrando que a promoção da saúde é o principal motivo pelo qual os idosos realizam atividades físicas. 
Neste estudo, assim como no de outros autores (LOUREIRO, 2007; DANTAS et al., 2015; RIBEIRO et al., 2012) existe uma representatividade massiva de mulheres em detrimento dos homens, no que se refere à participação em programas AF.

O processo de envelhecimento ao longo dos anos gera limitações físicas que influenciam a capacidade funcional dos idosos na realização das tarefas diárias, assim, entende-se que a dimensão saúde é a principal fonte de motivação para a prática de atividade física, pois o idoso tende a evitar o seu envelhecimento funcional e, com isso, manter sua qualidade de vida (MAZO; LOPES; BENEDETTI, 2009; CHAO; FOY; FARMER, 2000; PHILLIPS; SCHNEIDER; MERCER, 2004). No presente estudo os dados apontam para uma conscientização dos idosos sobre a importância da prática de AF, mesmo que ainda com lócus de controle externo (benefícios na saúde), caracterizando uma motivação extrínseca para a prática da atividade física. Em muitos casos, a recomendação médica também assume um fator externo de motivação, uma vez que profissionais da área da saúde exercem grande influência na tomada de decisão de idosos, orientando-os em relação aos fatores de risco e doenças e como minimizar tais problemas (OKUMA; MIRANDA; VELARDI, 2007).

Os resultados do presente estudo são extremamente importantes para profissionais que desenvolvem programas de AF com idosos. Visto que as atividades devem ser planejadas seguindo os princípios do treinamento, como da sobrecarga, para promoverem melhoras graduais nas capacidades físicas dos indivíduos idosos. A permanência em um programa de treinamento que não promova melhoras nas capacidades físicas, e, consequentemente, na qualidade de vida dos indivíduos, poderá desmotivá-los da prática.

A dimensão prazer revelou ser o segundo fator motivacional, apresentado pelos idosos para a prática de atividade física neste estudo. De acordo com a Teoria da Autodeterminação - TAD (DECI; RYAN, 1985), o prazer está inserido no contexto da motivação intrínseca, em que o sujeito possui plena satisfação na atividade (DECI; RYAN, 2000). Além disso, a TAD aponta que, quanto mais autodeterminada é a motivação, maior o tempo de permanência na atividade. Dessa forma, o prazer se torna importante para manter os idosos na prática de atividade física (TEIXEIRA et al., 2012).

Com essa motivação, que caracteriza um lócus de controle interno (motivação intrínseca), existindo uma melhora na autoestima do idoso poderá fazer com que ele permaneça na prática de atividade física por mais tempo (MEURER; BENEDETTI; MAZO, 2011). E ainda, a possibilidade de fazer atividades com outras pessoas e se sentir pertencente a um contexto social (sociabilidade) 
atrelado ao prazer são elementos que, quando levados em consideração, podem tornar o programa de atividade física ainda mais bem-sucedido.

Já a competitividade foi a dimensão que menos motivou os idosos à prática da atividade física. A dimensão atende para motivação intrínseca, pois se caracteriza como fator de regulação interna. Dantas et al. (2015) também observaram em seu estudo que contou com a participação de 39 idosos com idade média de $70( \pm 7,8)$ anos, praticantes do Método Pilates, a menor pontuação na competitividade no que tange a motivação à prática de atividade física em idosas.

O estudo de Ryan et al. (1997) aponta que a motivação extrínseca é importante para se iniciar a prática de atividade física, porém possui baixa relação com a sua continuidade. Dessa maneira é importante fazer com que a motivação para a prática de atividade física seja desenvolvida de forma autônoma. Fica evidente que os idosos não praticam atividades físicas pensando em vencer, ou adquirir sensação de superioridade e destaque em alguma atividade/desporto.

\section{Conclusão}

Com base nos dados aqui apresentados os idosos participantes do projeto universitário continuam motivados a praticar atividade física no mesmo local há dez anos ou mais, principalmente pelo aspecto de promoção da saúde, seguido pelo prazer e pela sociabilidade.

Quanto à importância que os idosos dão à prática da atividade física foi identificado que os mesmos têm ciência dos benefícios da prática voltada à manutenção da saúde, motivando-os a permanecerem no projeto, pois desta forma estariam melhorando sua qualidade de vida. E o principal aspecto citado pelos participantes da amostra referente à importância da atividade física foi novamente a concepção ligada à saúde com a prevenção de doenças ou a manutenção da saúde. Aspectos sociais e psicológicos também devem ser relevados, visto que a companhia de amigos é considerada importante pelo grupo de idosos.

Estudos sobre os fatores motivacionais de idosos para a prática de atividade física são extremamente importantes. De acordo com os pressupostos teóricos da TAD, quanto mais autodeterminado estiver um comportamento, mais próximo da motivação intrínseca estará e, desse modo, maior o engajamento e a aderência com a atividade proposta (RYAN et al., 1997; DECI; RYAN, 2000).

Mais programas voltados para atender a população idosa deveriam ser criados e/ou mantidos, tendo em vista o crescente número de idosos na 
população, gerando maior demanda no atendimento ao indivíduo idoso e a necessidade de políticas públicas de lazer, saúde e educação. Com novos programas, mais pessoas poderiam se beneficiar da prática de atividade física, contribuindo para um envelhecimento mais saudável, diminuindo seus principais sintomas negativos, tanto biológicos quanto psicológicos, e melhorando a qualidade de vida de seus usuários.

Se faz necessário dar continuidade nas investigações dos motivos que levam os indivíduos idosos a se comprometerem com a manutenção da prática de atividade física sob diferentes perspectivas, com amostras maiores, diferentes modalidades e em diferentes contextos na busca de maiores informações na formulação, organização e planejamento de programas para estes indivíduos.

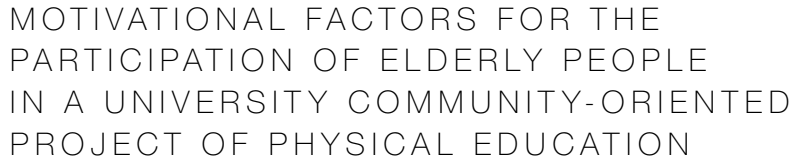

\section{abstract}

Objective: the study had as an objective to investigate factors related to the motivation of elderly people towards the practice of physical activity. Method: it is a cross-sectional descriptive study with 35 elderly people engaged for 10 years or more in a university social project. Data was gathered by conducting personal interviews regarding socio-demographics, practice of physical activity and motivation to take part in the project. In order to mensurate motivation the "Inventário de Motivação à Prática Regular de Atividade Física 54" (IMPRAF 54) was applied. Results: the sample was, by a large majority, composed of women ( $n=33,94 \%$ ) with mean age of \pm 74.05 (SD \pm 6.30 ) years. Results indicate that the elderly still motivated to practice physical activity mainly due to health related aspects, followed by pleasure and sociability. Conclusion: regarding the importance given to physical activity, it was identified that the elderly acknowledge its benefits, which makes them to remain in the program. The elderly stated that physical activity is related to disease prevention and/or health maintenance - in their conception, physical activity is intimately connected to health.

keywords

Motivation. Elderly. Aging. Motor Activity. Health Promotion. 
AFONSO, Mariângela et al. Do diagnostico à ação: Núcleo de Atividades para a Terceira Idade (NATI) - trajetória e construção. Revista Brasileira de Atividade Física e Saúde, Florianópolis, v. 15, n. 3, 2010.

BARBOSA, Marcus; BALBINOTTI, Marcos. Inventário de Motivação à Prática Regular de Atividade Física (IMPRAF-126). Porto Alegre: Laboratório de Psicologia do Esporte da Universidade Federal do Rio Grande do Sul, 2006.

CARDOSO, Amanda. Fatores motivacionais de idosos associados à prática regular de exercícios físicos. 2014. 122 f. Dissertação (Mestrado) - Instituto de Biociências de Rio Claro, Universidade Estadual Paulista, 2014. Disponível em: http://hdl.handle. net/11449/108758. Acesso em: 10 out. 2018.

CAVALLI, Adriana et al. Motivação de pessoas idosas para a prática de atividade física: comparativo entre dois programas universitários - Brasil e Portugal. Revista Brasileira de Geriatria e Gerontologia, v. 17, n. 2, p. 255-264, 2014.

CHAO, Dinnie; FOY, Capri; FARMER, Deborah. Exercise adherence among older adults: challenges and strategies. Contemporary Clinical Trials, New York, v. 21, n. 5, p. 212S-217S, 2000.

COSTA, Israel et al. A importância da atividade física para manutenção da saúde e os principais fatores que motivam professores, alunos e funcionários de duas universidades a praticarem exercícios. Revista Brasileira de Atividade Física e Saúde, Florianópolis, v. 8, n. 1, p. 52-61, 2003.

DANTAS, Ricardo et al. Fatores motivacionais de idosos praticantes do método Pilates. Revista Brasileira em Promoção da Saúde, Fortaleza, v. 28, n. 2, 2015.

DECl, Edward; RYAN, Richard. Intrinsic and extrinsic motivations: classic definitions and new directions. Contemporary Educational Psychology, New York, v. 25, n. 1, p. 54-67, 2000.

DECl, Edward; RYAN, Richard. Motivação Intrínseca e Autodeterminação no Comportamento Humano. Nova York: Plenum, 1985.

DUMITH, Samuel; GIGANTE, Denise; DOMINGUES, Marlos. Stages of change for physical activity in adults from Southern Brazil: a population-based survey. International Journal of Behavioral Nutrition and Physical Activity, New York, v. 4, n. 1, p. 25, 2007.

FRANCHI, Kristiane; JUNIOR, Renan. Atividade física: uma necessidade para a boa saúde na terceira idade. Revista Brasileira em Promoção da Saúde, Fortaleza, v. 18, n. 3, p. 152-156, 2005.

FREITAS, Clara et al. Aspectos motivacionais que influenciam a adesão e manutenção de idosos a programas de exercícios físicos. Revista Brasileira de Cineantropometria e Desempenho Humano, Florianópolis, v. 9, n. 1, p. 92-100, 2007.

KING, Abby. Interventions to promote physical activity by older adults. Journal of Gerontology, v. 56, n. 2 (Especial), p. 36-46, 2001.

LOUREIRO, Luciano. Motivação na prática de atividade física para pessoas na terceira idade. In: SEMINÁRIO INTERMUNICIPAL DE PESQUISA, 10.; MOSTRA DE ATIVIDADES EXTENSIONISTAS EPROJETOS SOCIAIS, 5.; SALÃO DE INICIAÇÃO CIENTÍFICA ETRABALHOS ACADÊMICOS, 8., 2007. Anais [...]. Canoas: Editora da ULBRA, 2007. p. 1-22.

MAZO, Giovana; LOPES, Marize; BENEDETTI, Tânia. Atividade Física e o Idoso: concepção gerontológica. Porto Alegre: Sulina, 2009.

MEURER, Simone; BENEDETTI, Tânia; MAZO, Giovana. Teoria da autodeterminação: compreensão dos fatores motivacionais e autoestima de idosos praticantes de exercícios físicos. Revista Brasileira de Atividade Física \& Saúde, Florianópolis, v. 16, n. 1, p. 18-24, 2011. 
MEURER, Simone; BENEDETTI, Tânia; MAZO, Giovana. Fatores motivacionais de idosos praticantes de exercícios físicos: um estudo baseado na teoria da autodeterminação. Estudos de Psicologia (Natal), Natal, v. 17, n. 2, p. 299-303, 2012.

MINISTÉRIO DA SAÚDE (MS). Vigilância de fatores de risco e proteção para doenças crônicas por inquérito telefônico (Vigitel). Brasília, DF, 2007. Disponível em: http://portal. saude.gov.br/portal/arquivos/pdf/vigitel2007_final_web.pdf. Acesso em: 12 maio 2017.

OKUMA, Silene; MIRANDA, Maria; VELARDI, Marília. Atitudes de idosos frente à pratica de atividades físicas. Revista Brasileira de Ciência e Movimento, Águas Claras, DF, v. 15, n. 2, p. 46-54, 2007.

ORGANIZAÇÃO PAN-AMERICANA DA SAÚDE (OPAS). Envelhecimento ativo: uma política de saúde. Brasília, DF: Organização Pan-Americana de Saúde, 2005.

PHILLIPS, Edward; SCHNEIDER, Jeffrey; MERCER, Greg. Motivating elders to initiate and maintain exercise. Archives of Physical Medicine and Rehabilitation, New York, v. 85, n. 3, p. 52-57, 2004.

PROCHASKA, James; DICLEMENTE, Carlo; NORCROSS, John. In search of how people change: applications to addictive behaviors. American Psychologist, Washington, DC, v. 47, n. 9, p. 1102-1114, 1992.

RIBEIRO, José et al. Adesão de idosos a programas de atividade física: motivação e significância. Revista Brasileira de Ciências do Esporte, Porto Alegre, v. 34, n. 4, p. 969-984, 2012.

RYAN, Richard et al. Intrinsic motivation and exercise adherence. International Journal of Sport Psychology, Roma, v. 28, n. 4, p. 335-354, 1997.

SAMULSKI, Dietmar. Psicologia do esporte. $1^{a}$ ed. São Paulo: Editora Manole Ltda, 2002.

SIQUEIRA, Fernando et al. Aconselhamento para a prática de atividade física como estratégia de educação à saúde. Caderno de Saúde Pública, Rio de Janeiro, v. 25, n. 1, p. 203-213, jan. 2009.

TEIXEIRA, Pedro et al. Exercise, physical activity, and self-determination theory: a systematic review. International Journal of Behavioral Nutrition and Physical Activity, New York, v. 9, n. 1, p. 78, 2012.

THOMAS, Jerry; NELSON, Jack; SILVERMAN, Stephen. Métodos de pesquisa em atividade física. Porto Alegre: Artmed Editora, 2009.

WORLD HEALTH ORGANIZATION (WHO). Global Strategy on Diet, Physical Activity and Health. Geneva: WHO, 2010. Disponível em: http://www.who.int/dietphysicalactivity/ publications/9789241599979/en/. Acesso em: 12 maio 2017. 
